\title{
A Comparative Analysis of Expert Opinions on Artificial Intelligence: Evolution, Applications, and Its Future
}

\author{
Falguni Saini, Tanya Sharma*, Suman Madan
}

Jagan Institute of Management Studies, Rohini Sec-5, New Delhi, India

* Corresponding Author email: tanyaa.sharma1902@gmail.com

\section{Article History}

Received: 30 April 2021

Revised: 28 August 2021

Accepted: 15 September 2021

Published: 28 September 2021

\author{
Student(s) \\ - Falguni Saini \\ - Tanya Sharma \\ Academic Year: 2020-2021 \\ Course Level: Bachelor \\ Course Name: BCA \\ Course year: $3^{\text {rd }}$ Year $/ 6^{\text {th }}$ Semester
}

Mentar(s)

- Dr. Suman Madan

\begin{abstract}
Artificial Intelligence (AI) is a field of computer science that primarily focuses on automating tasks that explicitly require human intelligence. The mechanics of AI technology majorly revolves around central affairs including knowledge representation, learning, problem-solving, reasoning, etc. Additionally, each discipline of AI focuses on a particular component to efficiently train the machines. Every branch of AI technology exploits knowledge in machines using diversified practices but with a clear idea of achieving the desired output. AI has evolved drastically over the past two decades and is considered the most in-demand technology at present times in varied fields including healthcare, education, forecasting, security, etc. This paper provides an extensive survey on artificial intelligence and related work going on in this field, how it differs from human intelligence, various subfields of AI and their importance, various issues related to AI and possible solutions along with and future trends related to this technology depicting people's reliability on it and various possible concerns.
\end{abstract}

Keywords: Artificial Intelligence, Human Intelligence, Machine Learning

\section{Introduction}

The capacity to think and analyze with the appropriate logical understanding of the subject for problemsolving can be possibly termed as intelligence. However, intelligence includes both human intelligence as well as artificial intelligence. Human intelligence refers to the intellectual power of humans that is marked by cognitive parameters, wherein artificial intelligence is the intelligence demonstrated by machines. Artificial Intelligence (AI) refers to the field of computer science that deals with the simulation of human intelligence in machines and enabling them to automate tasks. The primary goal of AI is to let computers exhibit human-like thinking capabilities by deploying knowledge in it through various tools, techniques, and algorithms. The evolution in the field of AI has surely made enormous advancements in the human world with dramatic revolutionary theories and techniques [1][2][3]. For instance, the AI-based four frontrunners; Google Assistant, Alexa, Siri, and Cortana have made the biggest possible impact in the daily lives of humans by providing efficient voice assistance to humankind. However, there is more to AI including selfdriving cars, the facility of personalized shopping experience in e-commerce, traveling miles with the aid of a reliable GPS, diagnosis of diseases using machines or robots, harvesting crops with the assistance of AI 
bots, sentiment analysis, and digital analytics on social media, processing on enormous amounts of data and much more.

Artificial Intelligence is not a newfound terminology or technology in the world of researchers. It is believed that roots and traces of AI could also be found in Greek mythology.[2] However, the term "Artificial intelligence" was officially used and adopted by John McCarthy in 1956 at the Dartmouth Conference. AI has undergone various scientific and technological developments over the past few decades which entailed varied transitional boom periods for this technology [4]. The advent of neural network software along with significant techniques that proved a considerable number of mathematical theorems correctly in the 1950s signalled the dawn of artificial intelligence and claimed it as the birth era of AI. Furthermore, during the late 1970s, the emergence of expert systems and speech recognition led to the boom period of AI [4]. Henceforth, the introduction to machine learning and deep learning algorithms around the late $90 \mathrm{~s}$ and early 2000s respectively made AI, the most in-trend and ruling technology even until the present times. AI has generously evolved over the years and has undoubtedly affixed itself to human life in almost all aspects. In today's times, machines can even surpass human intelligence in some areas and are capable enough to make decisions using the exploited knowledge and experience, without human intervention. The gained popularity of $\mathrm{AI}$ is also due to the ease it has provided to the entire mankind by automating tasks that are sometimes considered taxing to humans, mentally as well as physically [5][6].

We are optimally utilizing current AI technology and continue to make progress towards creating more powerful and innovative AI techniques that can help us to create more reliable and effective AI systems to cater to the needs of billions. AI is indeed a tool with endless opportunities at its disposal and if the correct steps are taken to shape the future, then it can be used as a counter tool to manage the ill-effects of a broad variety of technological advancements, climate change, and many more. When there have been major advancements in providing human-like intelligence to machines, many AI researchers now aim to design a better, more evolved artificial intelligence if it can be created.

In recent years, lots of work has been done in the area of artificial intelligence. J. Shabbir et al. [1] discussed the relentless growth of AI and how it majorly differs from human intelligence. The authors discussed how AI cannot be entitled as a purely clever and intellectual model as humans due to a lack of emotions. However, the spatial references that are provided by the AI techniques are built to show how the reference frame needs to be established. Furthermore, the challenges of AI that directly impact society including factors like security, verification, validity, and control are discussed with possible solutions. The future predictions of AI enlist increased profitability and economic growth rates in many industries. Various strategies such as placing human factors to the central nucleus, AI agents exhibiting human emotions, etc. can prove to be revolutionizing advancements in the technological world. R. H. Ner, et al, [2] discusses the two major categories of AI. Conventional AI, also known as good-old-fashioned AI is subdivided into methods including expert systems and behaviour-based AI. Another category includes Computational Intelligence $(\mathrm{CI})$ which further includes methods like neural networks, fuzzy systems, and evolutionary computation. It is concluded that AI works as an artificial human brain with an unbelievable computational capacity. A. R, et al, [7] discusses the importance of central problem traits of AI in the efficacy of intelligent agents along with the varied approaches and techniques of AI. It majorly highlights how AI can alleviate the efficiency in software engineering by error recognition and correction with the assistance of expert systems. AI has its fair share of pros and cons which tells us to be readily prepared for revolutionary changes shortly due to explicit advancements in this specific field. However, the infinite possible capabilities of the machines are an indication for the programmers to work responsibly to prevent major accidents. J. Borana, et al. [8] divides AI into three major categories including cognitive science applications, robotics applications, and natural interface applications. It aims to highlight the extensive difference between weak AI and strong AI. Strong AI prospects machines with the same computational capabilities as humans while weak AI implies adding cognitive feats to the machines to make them useful tools to the human force. However, the Turing test is the basis of measuring the effectiveness of an AI model. In the future, AI will 
widen its arena in all application fields. Therefore, the major goal of AI relies on creating machines that can even surpass human intelligence.

H. Lu, et al, [9] proposed that current developments in AI are mainly focusing on ICT- Information communication technology and RT-Robot technology but these current developments lack many aspects of human intelligence such as the ability to multitask, have self-motivation, self-understanding, and selfconsciousness among many more. Therefore, It focuses on developing an intelligent learning model called "Brain Intelligence" which would be able to learn from virtual scenarios without having to experience them in real life by making use of an imaginary function. It shows how BI models can solve many existing problems in $\mathrm{AI}$ such as frame problems, the association function problem, and symbol grounding problems. J. Lui, et al, [10] analyses the trends in references stated in AI journals and research papers and found a decrease in self-references indicating growth towards open-minded AI. The paper also explored various topics of research in AI over the years and found an inner connection among these topics as well as hot topics for conferences that differ from those in journals. J. Shaw, et al, [11] proposed use cases of machine learning- a field of AI by presenting a framework to help enable its use in the healthcare industry such as by giving decision support and automation. However, challenges such as privacy, consent, algorithmic bias, security, scalability, the changing nature of health care workers, etc. continue to exist. It suggests that the future of $\mathrm{ML}$ in health care may prove beneficial but is uncertain as it depends on the support of patients, health care workers, and stakeholders. R. Dasoriya, et al, [12] discussed various issues regarding the development and future of AI such as its adverse effects on society along with the benefits it offers. It suggests that despite offering various advantages such as easier fraud detection, efficiency, space exploration, and substituting humans in performing dangerous tasks, the future of AI remains uncertain due to the risk of high unemployment rates, lack of ethics and other virtues, high maintenance, and repair cost to cope up with changes in real-world, etc. making the future of AI debatable.

\section{Artificial Intelligence vs. Human Intelligence}

AI can be divided into two major categories: Strong AI and Weak AI. Strong AI is a form of AI that can act as humans efficiently without much scope for errors. It consists of machines that have human intelligence which allows them to think, reason, and perform activities like humans. Strong AI has not yet been developed as current research is incapable of providing us with such advanced technology. On the other hand, weak AI has made quite some progress in real-world scenarios. It aims to create machines that are capable of thinking in some scenarios and deciding their next move such as playing chess or ludo against a computer system powered by AI. This application does not make use of much planning and thinking by the computer but it simply follows a set of instructions fed into the machine by a human. A test called the Turing test is used to measure the reliability of $\mathrm{AI}$ techniques to determine the best available technique to use in real-world scenarios for optimum results [8].

Human intelligence is the intellectual power of humans consisting of cognitive feats such as the ability to think, learn, analyze, understand, innovate, react and make use of the acquired knowledge for decisionmaking, problem-solving, or manipulation of objects. Wherein, Artificial Intelligence refers to the potential of machines to perform automated tasks that require logical thinking and decision-making for problemsolving, without human intervention. [7] Thus, human intelligence is analogous whereas artificial intelligence exhibits digital behaviour as humans use their brain's intellectual power for processing information but AI depends on data and specific information to be fed into the system. AI is used to develop and train machines or robots to adapt human behaviour and characteristics including the capacity to learn from past experiences, sense, predict and determine the meaning of a certain situation. At present times, AI tools are widely used in various sectors including schools, hospitals, industries, military, telecommunication, entertainment, quantum science, agriculture, sports, travel, and many more.[5] Precisely, artificial intelligence is the derivative of human intelligence but AI has taken over a massive dimension and is believed to be challenging natural intelligence in distinct ways globally.[4] Despite having made enormous advancements in the technological environment, AI still differs from human intelligence in factors like lack 
of emotions, experiencing zero fatigue even after working for hours continually, exceptional computational speed, disability to combat multifaceted circumstances, and impotent to invent.

Indeed, even clearly drastically unique AI frameworks have numerous normal methods. Four significant ones are:

- Representation of knowledge: It should be addressed in some way or another - maybe as a series of on the off chance that standards, as a casing-based framework, as a semantic organization, or in the association loads of an artificial neural network.

- Learn from knowledge: Taking in Automatically developing information from the climate -, for example, obtaining the standards for a standard-based master framework, or deciding the proper association loads in a counterfeit neural organization.

- Rules Formation: These could be unequivocally incorporated into a specialist framework by an information engineer, or certain in the association loads learned by a neural network.

- Searching: This can take numerous structures - may be looking for a grouping of states that drives rapidly to an issue arrangement, or looking for a decent arrangement of association loads for a neural organization by limiting wellness work.

\section{Major Disciplines of AI}

This section highlights the subfields related to AI using a variety of techniques, as shown in figure 1 . AI has made a permanent spot for itself in the world of technology. Natural Processing Language (NLP), Expert Systems, Robotics, Machine Learning (ML), and Artificial Neural Networks (ANN) are key discipline areas of AI that are broadly used for innovations and enhancement in its arena.[2].

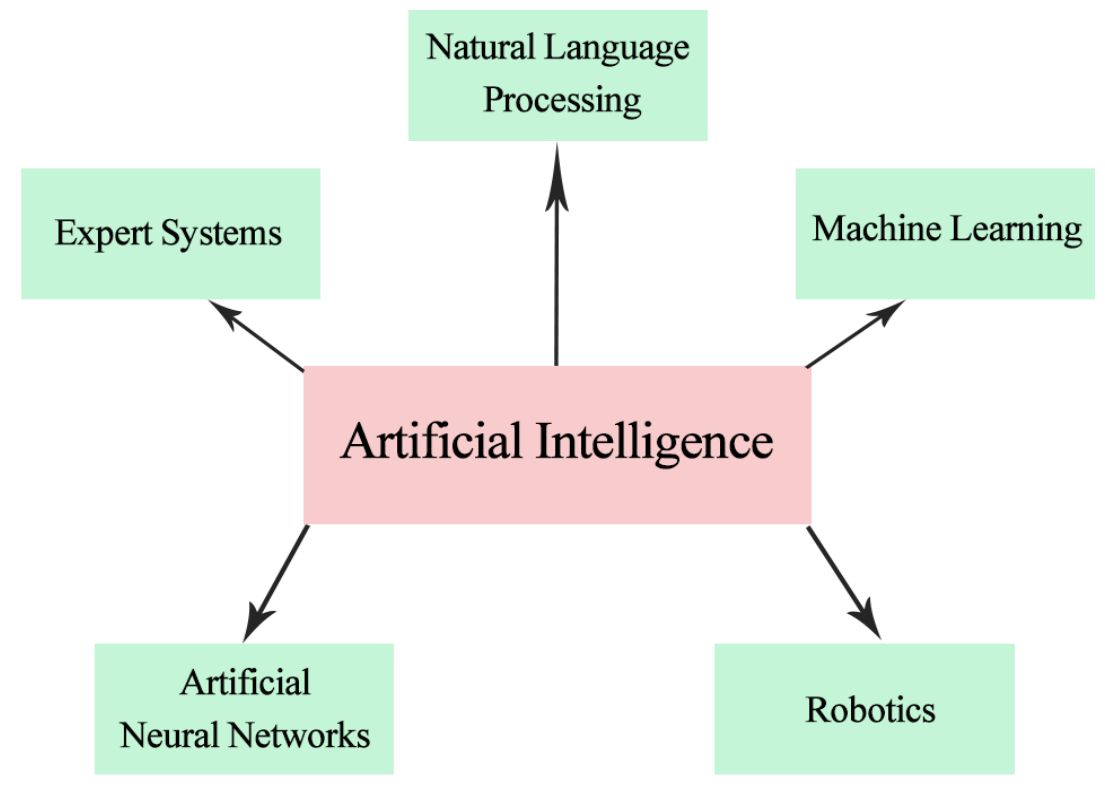

Figure 1: Disciplines of Artificial Intelligence

\subsection{Natural Language Processing}

Natural Language Processing is a subfield of Artificial intelligence, computer science, and linguistics that is concerned with the ability of computers to read and understand languages that humans speak. NLP relates to the interactions between human beings and machines. In this technology, computers are programmed in a way to process and examine enormous amounts of natural language data. It also includes information retrieval and machine translation.[3] NLP finds its applications in areas like text classification, information mining, chat bots, virtual assistants, spelling amendments, syntax checking, speech recognition, sentiment 
analysis, etc. For instance, Amazon uses the NLP techniques for the interpretation of customer reviews; Twitter uses NLP to filter the terroristic language from the tweets; the auto-correct feature on various platforms is a major contribution of NLP.

In a practical approach, NLP libraries are used by programmers to teach machines how to extract the relevant information from the available text data. For example, NLP is used in spam detection as it can identify junk emails by evaluating the text of the subject or body content.

\subsection{Expert Systems}

Expert System is a subfield of AI that possesses the capability of centralized decision-making with the same intellectual computational capability as a human expert. These systems are primarily designed to solve complicated problems in a particular domain using logical reasoning and knowledge. It uses symbolic representations to perform computations. Expert systems handle tremendous amounts of data and are profoundly responsive. For example, an expert system provides suggestions for correcting spelling and other errors in the Google search engine.

The effectiveness of an expert system directly relies on the expert's knowledge that is assembled in a knowledge base. The more information is accumulated in the knowledge base, the better is the efficiency of the expert system. The key components of expert systems are user interface, inference engine, and knowledge base; wherein the key participants in the development of AI expert systems are domain expert, knowledge engineer, and end-user respectively. This branch of AI holds its maximum application in automatic speech recognition, medical diagnosis, predictions, airline and cargo scheduling, virus detection, stock market trading, etc.

Few examples of AI expert systems:

- PXDES is a clinical support system to predict the degree as well as the type of lung cancer;

- CaDet is an effective expert system that can identify cancer at early stages;

- DENDRAL is used for chemical analysis to predict molecular structure,

- MYCIN is an expert system based on the backward chaining process which can efficaciously spot variable bacteria which can become a potential cause for acute infections.

\subsection{Robotics}

Robotics is an interdisciplinary branch of AI wherein the intelligent agents known as robots are mechanically designed to operate in the actual physical world. The main objective of this branch of AI is to design robots that can accomplish tasks with absolute accuracy that were originally done by human beings only. Robots have made it easier to necessitate activities that were monotonous and possibly dangerous if not carried out with full accuracy for humans. [13]. Robots are deployed for various machining processes, packaging of finished goods in industries; intelligent agents like Da Vinci Robot are used to assist doctors in performing complicated surgeries; robots have become the best suited option for space exploration, for example, Mars Rover is an autonomous robot that travels to Mars and clicks pictures of the Martian rock formations; robotic devices can be used in agricultural sector for harvesting and removal of weeds; remotecontrolled robots are sent into depths of oceans for underwater exploration and collecting data and images about the species; drones are used in military for surveillance on enemies, for example, a tactical combat robot named as DOGO has a spying camera and a pistol for emergency circumstances, humanoid robots like Nadine, Junko Chihira looks exactly like a human being and are used for customer service agenda wherein these robots can identify a customer based on their earlier visits, make eye contact, shake hands and chat with the customers.

\subsection{Machine Learning}

Machine Learning is a branch of artificial intelligence that learns using the fed data and improves based on experience. It identifies various patterns to acquire knowledge and performs tasks on its own without being explicitly programmed. In a general sense, it is the science that empowers machines to decipher, execute 
and examine information for tackling real-world problems. With the deployment of complex numerical ability, software engineers plan machine learning calculations that are coded in a machine dialect to sometime create a total ML framework. In this way, ML enables us to perform assignments to classify, translate and assess information from a given dataset. It fundamentally merges on the applications that adjust from involvement and progress their decision-making potential or precision over some time. Image processing, speech recognition, classification, association, regression, extraction, prediction, statistical analysis is some of the major real-life application areas of machine learning. Despite heavy traffic, Google maps direct you on to the fastest route; automatic friend tagging suggestion using image processing and face detection on social media platforms; product recommendation through ads; dynamic pricing by tracking buying patterns, etc. are all a contribution to society by machine learning itself.

\subsection{Artificial Neural Networks}

Artificial Neural Network aims to simulate the working of the biological human brain by using silicon and wires. ANN is a structured computational network and the interconnected nodes are referred to as artificial neurons. It works on the belief that microchips can imitate the behavioural characteristics of neurons and dendrites. ANN sights at complex problem-solving efficiently that would prove quite difficult for humans. ANN possesses parallel processing capabilities. Information that's utilized in conventional programming is stored on the entire network, not on a database. The vanishing of some pieces of information from one place doesn't prevent the network from working. After ANN preparation, the data may yield output even with lacking information. The loss of performance here depends upon the centrality of lost data. ANN is utilized in areas like speech and character recognition, stock market prediction, image compression, semantic parsing and question answering, paraphrase detection, forecasting, etc.

\section{Central Issues Related to AI and Possible Solutions}

\subsection{Reasoning, Problem Solving}

Human beings supposedly put forward an intrinsic logical judgment based on the acquired knowledge, be it playing board games, performing mathematical calculations, solving puzzles and riddles, etc. However, reasoning requires the brain's explicit computational capability for solving complex problems.[7] AI also adopts a somewhat similar trait in its tools for better performance. It follows the step-by-step reasoning and logical explanation criteria in each task that requires problem-solving in a certain situation. In AI, machines are trained using various algorithms that subjectively hints towards logical reasoning.

\subsection{Knowledge Representation}

Knowledge representation is the foremost central aspect of AI. To perform any automated task, AI tools are expected to know the real world. This field is dedicated to representing information about the world to computer systems so that it can be utilized for solving complex problems extensively. AI needs to represent objects, properties, and relationships between the objects, different situations, events, causes, effects, and knowledge about the given knowledge [7]. It incorporates findings from psychology on how humans represent knowledge, interprets information, and give out a solid as well as logical evaluation.

\subsection{Planning}

AI planning is the branch of artificial intelligence that is majorly concerned with the realization of sequence actions and strategies. Intelligent agents must be able to set and achieve goals but the need to visualize the representation of the world's state and to be able to make predictions on how their actions will impact a certain situation is also necessary [7].

Planning is crucial for picking out the best choice and maximizing the utility of all the available choices. However, it is important to check whether the predictions match up to the world's actions and change the plan accordingly if necessary. 


\subsection{Learning}

Machine Learning has been the central aspect of AI research from the very beginning. Machine Learning or ML, is the study of computer algorithms that improve on their own through experience and use of fed data. It is considered a part of AI and basically, it involves enabling the machines to perform various tasks without any explicit programming. Machine learning is broadly divided into three categories depending upon their nature:

- Unsupervised learning is the learning algorithm in which it independently finds patterns through the input stream.

- Supervised learning is presented with example inputs and desired outputs, wherein the major goal is mapping inputs to the outputs. It includes both classification and regression.

- In Reinforcement learning, the computer program interacts in a dynamic environment in which it is expected to perform a specific task and the feedback is provided in terms of rewards.

\subsection{Perception}

Machine perception is the ability of computer systems to interpret data in such a manner that is similar to how human beings sense through their sensory organs to relate to the real world. The main goal of perception is to enable machines to see, feel and perceive the world around them as humans do. The basic method to equip perception is practically made possible through the machine hardware and software. Sensors (cameras, microphones, etc.) and input devices (keyboard, mouse) are used to deduce aspects of the world for perception. Computer vision, machine hearing, machine touch, object recognition, speech recognition, facial recognition, and machine smelling are a few sub-disciplines of machine perception [7].

Table 1: Comparison among various opinions on the Future of AI stated by experts

\begin{tabular}{|c|c|c|c|}
\hline Name & Opinion & Solution & Nature \\
\hline $\begin{array}{l}\text { Erik } \\
\text { Brynjolfsson }\end{array}$ & $\begin{array}{l}\text { The world will be a better place. Poverty } \\
\text { can be eliminated virtually, with less } \\
\text { disease and better education. But deadly } \\
\text { weapons may come into existence and } \\
\text { wealth and power may become excessively } \\
\text { concentrated. Neither outcome is } \\
\text { inevitable. }\end{array}$ & $\begin{array}{l}\text { Suitable measures should be } \\
\text { taken to ensure that technology } \\
\text { matches human values. This } \\
\text { needs to be done at all levels- } \\
\text { Government to individuals. }\end{array}$ & Neutral \\
\hline Bryan Johnson & $\begin{array}{l}\text { AI may lead to a situation where humans } \\
\text { become irrelevant - not only in the case of } \\
\text { jobs but existential irrelevance. }\end{array}$ & $\begin{array}{l}\text { Economic systems should focus } \\
\text { on improving human well-being } \\
\text { and cognition. }\end{array}$ & Negative \\
\hline $\begin{array}{l}\text { Michael } \\
\text { M. Roberts }\end{array}$ & $\begin{array}{l}\text { He believes that AI still has a lot of ways to } \\
\text { improve but with the improvement, it will } \\
\text { require more and more user data } \\
\text { compromising security to provide comfort. } \\
\text { He suspects that this tradeoff of comfort and } \\
\text { intrusion in users' privacy will continue. }\end{array}$ & $\begin{array}{l}\text { Suspicion is that this will } \\
\text { continue if AI continues to grow } \\
\text { at this rate. }\end{array}$ & Negative \\
\hline Daniel Obam & $\begin{array}{l}\text { AI will help humans in making better } \\
\text { decisions professionally. It will aid } \\
\text { authorities to prevent crimes and uphold the } \\
\text { law.AI would improve the way humans } \\
\text { work and travel also allowing authorities to } \\
\text { analyze historical data and learn better. }\end{array}$ & $\begin{array}{l}\text { Cybersecurity practices need to } \\
\text { be improved to be capable of } \\
\text { handling any misuse of AI. }\end{array}$ & Positive \\
\hline
\end{tabular}


A Comparative Analysis of Expert Opinions on Artificial Intelligence: Evolution, Applications, and Its Future

\begin{tabular}{|c|c|c|c|}
\hline $\begin{array}{l}\text { Andrea } \\
\text { Romaoli } \\
\text { Garcia }\end{array}$ & $\begin{array}{l}\text { She believes that AI can indeed help } \\
\text { humans make better decisions in various } \\
\text { industries by providing a multitude of } \\
\text { information but it is largely dependent on } \\
\text { rich countries, as poorer countries may not } \\
\text { have the resources to train individuals and } \\
\text { make effective use of AI which may result } \\
\text { in wars and invasions. }\end{array}$ & $\begin{array}{l}\text { Richer countries need to help the } \\
\text { poorer countries to adopt AI and } \\
\text { benefit from it. }\end{array}$ & Neutral \\
\hline $\begin{array}{l}\text { Andrew } \\
\text { McLaughlin }\end{array}$ & $\begin{array}{l}\text { AI has various short-term benefits and } \\
\text { comforts but also has many long-term } \\
\text { issues which may take decades to get } \\
\text { recognized by people. It may cause } \\
\text { problems with jobs and discrimination in } \\
\text { the performance evaluation of individuals. }\end{array}$ & $\begin{array}{l}\text { The long-term effects of } \\
\text { advancements in AI should be } \\
\text { carefully analyzed and } \\
\text { appropriate measures are taken. }\end{array}$ & Negative \\
\hline Charles Geiger & $\begin{array}{l}\text { AI may pose threats but democracy and free } \\
\text { press would allow humans to tackle any } \\
\text { such arising threats. }\end{array}$ & $\begin{array}{l}\text { Advancements in AI should not } \\
\text { hinder democracy or freedom of } \\
\text { the press in any way. }\end{array}$ & Positive \\
\hline David Wells & $\begin{array}{l}\text { Advancements in technology are } \\
\text { accompanied by fear and anxiety but as the } \\
\text { pace of advancements has increased, people } \\
\text { need to adapt more quickly to harness the } \\
\text { benefits of newer technologies. AI will be } \\
\text { immensely helpful in providing better } \\
\text { quality education, medical support, and a } \\
\text { decrease in crime rates will improve the } \\
\text { quality of life for everyone. }\end{array}$ & $\begin{array}{l}\text { People need to understand the } \\
\text { benefits that AI offers and adapt } \\
\text { accordingly, helping humankind } \\
\text { extract its benefits and make } \\
\text { amends to its faults. }\end{array}$ & Positive \\
\hline $\begin{array}{l}\text { James Scofield } \\
\text { O'Rourke }\end{array}$ & $\begin{array}{l}\text { The future effects of AI depend on its } \\
\text { applications. It may lead to disasters or may } \\
\text { assist humans to achieve greater success, all } \\
\text { depending on its usage. }\end{array}$ & $\begin{array}{l}\text { It is necessary to make sure that } \\
\text { humans have control over } \\
\text { advanced AI and not vice-versa. }\end{array}$ & Neutral \\
\hline Marina Gorbis & $\begin{array}{l}\text { AI may lead to greater economic } \\
\text { inequalities. Whenever humans program } \\
\text { their environment, they also program their } \\
\text { interactions which lead to more non- } \\
\text { human-centric interactions. }\end{array}$ & $\begin{array}{l}\text { Humans need to become more } \\
\text { standardized and remove } \\
\text { vagueness from their } \\
\text { interactions by careful research. }\end{array}$ & Negative \\
\hline $\begin{array}{l}\text { Aneesh } \\
\text { Aneesh }\end{array}$ & $\begin{array}{l}\text { AI would automate tasks that require } \\
\text { human interaction and negotiation which } \\
\text { will lead to less socially active individuals } \\
\text { causing loneliness and depression among } \\
\text { people. The United States is already } \\
\text { witnessing this effect. }\end{array}$ & $\begin{array}{l}\text { Not automating tasks requiring } \\
\text { social interaction is necessary } \\
\text { for human well-being. }\end{array}$ & Negative \\
\hline
\end{tabular}

By these opinions (Table 1), it is clear that there exist different opinions about the advancement of Artificial Intelligence. AI has immense benefits to offer to mankind but it is also largely dependent on its use. It can lead to a constructive or a destructive future depending upon whether appropriate actions to control, manage and distribute it are efficiently taken or not. Out of 979 respondents, most of the people expect a positive outcome from the adoption of $\mathrm{AI}$ and making use of it with related technologies to serve humanity 
Saini et al., Adv. J. Grad. Res.; Vol. 11, Issue 1, pp: 10-22, January 2022

in a better way but many of them believe it may have a negative impact and appropriate measures need to be taken to control its power. 25 people denied choosing any of the options as shown in Fig. 2.

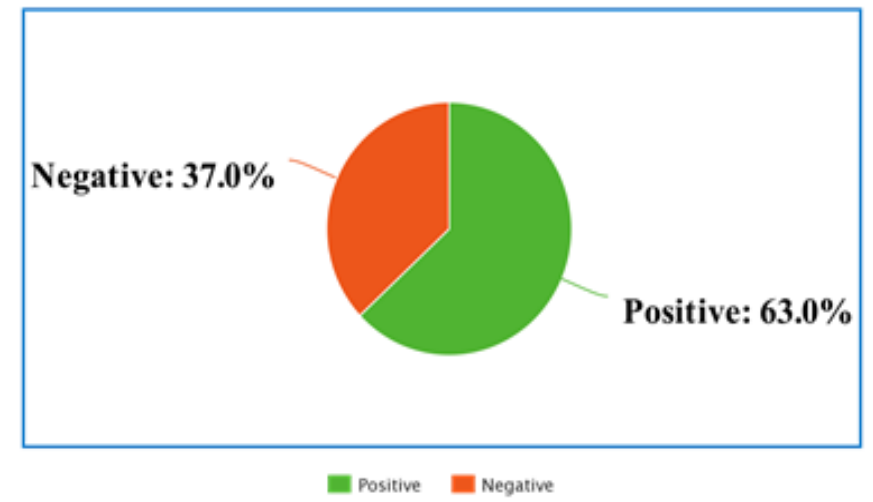

Figure 2: Nature of Response

The survey was conducted by Pew Research Center and Elon University's Imagining the Internet Center in July-August 2018. The respondents consisted of 10,000 experts and members of the interested public.[14] These opinions majorly highlight people's concerns about humankind being dominated by AI if not controlled properly which makes it important for authorities as well as individuals to take appropriate measures to ensure a safer future and harness all the benefits that AI provides to enhance the working of industries such as medical research and treatments, businesses, financial markets, agriculture, transport, etc.

\section{Application Areas}

In recent times, AI has spanned over vast and distinct domains. Few of the applications are discussed in this section.

\subsection{Network Intrusion Detection Systems (IDS)}

Intrusion Detection Systems are used to monitor and protect communication networks from potential intruders. It is vital for an organization's security. It becomes difficult for large organizations to monitor the large amount of data that flows through their network but delay in reporting the threats or worse, being unable to prevent such attacks can dramatically affect an organization's working and clients. Therefore, AI techniques and ML algorithms are now being used to automate this task or assist the technicians in finding faults or anomalies on the network which prove to be fatal if left unresolved. Machine learning (ML) makes use of algorithms that learn from data and then use that learning to make future predictions. These algorithms are trained using large amounts of data that enable them to predict results on future data sets. The data sets that are fed to the algorithms to train them are known as training sets. Machine learning algorithms can be first trained on sets of network data that are considered to be normal traffic and do not pose any harm. Once enough data is fed into these algorithms, they are ready to be used in the real world. A lot of false positives may be reported by the algorithm if the training set was faulty so it is necessary to feed in the correct data set. When the algorithm is used on the network traffic, it would make use of its current knowledge to predict whether the incoming data is behaving normally or is deviated from the normal pattern and needs to be reported as malicious. Hence, ML which is a sub-field of AI is of great use in detecting network anomalies.

\subsection{Medical Area}

AI offers services that can be applied in almost all the medical fields such as synthesizing medicines, creating and helping to test new vaccines, diagnosis of diseases in patients, and helping in treatments. Specifically, AI has helped in Image classification used for diagnoses, measuring the extent of damage caused by disease improving the accuracy of treatment. Electronic medical records are now maintained in hospitals which paves the way for the usage of Natural Language Processing which can provide structured reporting and 
EMR classification and various data mining techniques can also be applied to analyze similarities in data to help in the treatment of future patients. Image recognition techniques can also be helpful in image registration, segmentation, and classification. Surgical robots built using Robotics which is also a sub-field of AI can prove to be beneficial and assists doctors in surgeries requiring preciseness that can easily be achieved by making use of AI-powered robots. Many fields in the medical area depend on analysis and pattern-finding in images and many doctors spend their time looking for such patterns to assist further research and treatment. Usage of image classification techniques and ML algorithms helps to automate a lot of routine work of doctors and in some cases, machines may even recognize minute patterns in images that may even get overlooked by doctors. Rule-based AI is also used in healthcare systems which are simply a collection of if-else statements which act as a decision support system and help maintain the accuracy of the decisions made in the least possible time. Human errors are largely reduced by the usage of simple programs and machines. ANN (Artificial Neural Networks) is a simulation of the human brain neurons and the way they are connected with each other and learn from experience. It is also a collection of neural units based on the biological neural network present in the brain. It is designed to work exactly like the human brain. ANN is used in the medical area to make rapid error-free decisions and they learn from the data provided. It saves a lot of time for the doctors and assists in creating a much more efficient healthcare system.

\subsection{Accounting Databases}

Maintaining databases is a complex task when handling large amounts of data and dealing with critical information. Humans often have to work very hard to analyze the data. Integrating AI techniques have made it easier for humans to manage large amounts of data and mitigate problems. AI tools and techniques assist in searching, sorting, and evaluating data in real-time. In recent years, developments in AI have changed accounting systems. Almost all the accounting processes have been automated and organizations need IT skills more than accounting skills as much part of the accounting and finance has been automated with the help of AI-powered systems and employees are required to work with these systems to maintain accurate accounting records. Due to the increased reliability and less cost of such systems, organizations are investing in Robot Process Automation (RPA) which helps in automating the accounting entries efficiently. Also, such systems are capable of handling large amounts of data and its gain intending to get processes at a faster speed with less or no errors, they are also capable of making unbiased, quick decisions whenever needed. They can detect weak and complex patterns in the accounting and finance system, thus providing better predictions of expected results of future works of the organization. They act as efficient decision-makers and assist in making financial decisions that are probable to yield the best results for the firm like predicting the firm's revenue which helps the firm to make financial decisions accordingly. These systems may also produce errors but their chances are low and whenever they make a decision whether correct or false, they tend to learn from it which increases their accuracy with usage. Accountants are using their technical skills and knowledge to assist businessmen and stakeholders to make better decisions. A lot of data analysis is required to come to a decision which is often interpreted from facts and figures in the data stored in the system. This data analysis may not always be financial but may also be non-financial such as user's choice trends and company's capacity to produce particular goods. When these basic tasks are taken care of by AI systems, it paves the way for accountants to work on more important tasks which require human interaction such as planning strategy, customer relations, etc. These systems also provide the ability to detect frauds easily as ML algorithms learn from data and find patterns in it that define "normal" and whenever any suspicious activity or transaction takes place, it triggers a flag which then assists the accountant to take the required action.

\subsection{Computer Games}

Technological advancements have provided us with an interesting way to pass our free time by playing games virtually. Over the years, the integration of $\mathrm{AI}$ in game-playing has taken games to another level. 
Many features and issues are resolved with the help of AI such as non-playing characters (NPCs) pathfinding, making decisions efficiently, automating replies to real users playing, and making the games look and feel more real. Many techniques are used to implement these features like Pathfinding, Fuzzy logic, Bayesian networks, etc. [15]. Nowadays, the games created are getting increasingly interactive to provide the best gaming experience to the users. Earlier, AI was only used to provide an enemy to play against the user but the applications of AI in game-playing have increased significantly. It makes use of AI to design NPC and also program their reactions dynamically based on the action of the user. It is also used to simulate human emotions in-game and respond to audio and video input by the user. Based on the user's playing strategy it may also suggest future actions which he/she may want to take next or certain games by the same developer that the user may like to play. AI-powered bots may also be used in games to complete the requirement of players which enable the users to play the game without having to wait for real players to join. This enhances user retention as fewer the users have to wait, more are the chances that they will keep playing. Also, many AI-based components of the game can be reused between games and platforms such as sentimental analysis, creation of real-looking NPCs with human gestures, behaviour, replies, and lipsyncing with said dialogues, automated changes in difficulty level, performance analysis of the player, etc. This saves developers' time so that they can focus on more specific and important functionalities for the game such as detailed graphics for a graphics-oriented game or speed/driving settings for a racing game etc.

\section{Future Prospects of AI}

In the 21st century, the advancements in the field of AI have seen major improvements to the extent that it is now assisting humans to make accurate, error-free decisions in various sectors of critical importance such as health and defence. Also, day-to-day activities of a major part of society are also made possible due to the use of AI such as in search engines, chat-bots, and digital companions such as Siri, Alexa, and Cortana. Although they are not as close to human intelligence as other applications of AI but are continually becoming more efficient and powerful. The immense developments in fields of Natural Language Processing (NLP), Machine learning, and Deep learning have aided in the development of AI as a whole, providing it with the capabilities to match or even exceed human's efficiency at performing some important repetitive tasks such as for manufacturing goods with great precision or detecting defective supplies.[5] Many software-based services such as Google search, digital assistants, games, energy, and industrial operations depend on the use of AI for executing its main operations. Along with all the advantages that the use of AI is providing us, various concerns about the privacy of users, security of data, and complete replacement of humans keep threatening the minds of people.[3] According to experts, a major part of the population lacks the resources needed to fully utilize AI technology leaving them unknown of its use and with no knowledge of its impact in the future. Many experts believe that AI can pose some potentially dangerous risks to mankind ranging from increased vulnerabilities to cybercrime and cyber warfare to AIcontrolled drones and missiles, designed to kill the enemy without considering other factors that only humans are capable of evaluating accurately. A few also fear that AI may take over human intelligence and may cause mass destruction. Users who make use of AI and provide it with some data may be exposing them to cybercrimes and some extent, their independence of choosing the right to privacy. People have been making use of AI to cater to their specific needs but are also thought to be giving some control of their lives to the digital world with the aim of getting their tasks done faster and gaining advantages of data storage, searching things, staying connected with people socially or to simply remain entertained. Also, many users sharing their data with AI-controlled apps are unaware of how their data may be used and affect their daily lives but they keep on choosing comfort over privacy [14].

Future prospects of AI are wide and enhancements in this field may contribute towards making human life easier but there are still debates going on as to whether this technology will provide more benefits to the human population or will cause more harm to it. Table 1 provides a summary of various experts related to AI and the possible solutions related to future aspects of AI. At Silicon Valley, research has made 
breakthroughs towards the possibility of creating "strong AI" or "full AI" which would enable machines to work just like humans and perform any necessary actions such as driving vehicles, cleaning the room, and helping the users in case of emergencies. There are also speculations that AI may develop to an extent where it learns to self-improve itself and humans would no longer need to create future generations of AI and this could cause dangerous and irreversible effects on humanity. Although it is believed that superintelligence may take decades or centuries to occur if it at all turns possible, there are still concerns about how safe it would be [16]. Various people, including experts in the AI field, have different opinions about the advancements being made in AI. A part of the population believes that advancements in AI will maximize profits for businesses, boosting the economy and nurturing business-customer relationships. It will also revolutionize the way industries work by providing them with a wide variety of options to expand their businesses and produce better quality goods and services for their consumers. Major changes in the field of agriculture, health, and medicine, manufacturing, warfare, and defence are expected as a result of enhancements made in this field. Robots empowered with the use of AI can be used to cater to agricultural needs, increasing production and their assistance in the medical field would help in creating faster and more boosting the economy and nurturing business-customer relationships avoiding delays and errors in manual work. The integration of AI with search engines will also ensure good quality information decreasing the response time and maintaining better customer retention, increasing profits [1][5][6][13]. Despite all the benefits, a major part of them believes that AI may provide biased benefits instead of giving everyone equal opportunities to better their lives. It is thought to cause an increase in the gap between rich and poor with only the elite getting the benefits from AI and leaving the rest in an even worse situation causing major issues to democracies around the globe. Even over the past decade, it has not been clear whether the development of AI has benefited the global population. It is believed that the enhancements in AI technology will leave humans with little control over their lives, from choosing breakfast to TV shows and traveling routes, everything will be chosen by AI systems to maximize efficiency and maybe maximize profits of big businesses funding these AI systems.[13] There is a possibility that AI may replace humans on a large scale, leaving a major part of people unemployed and thus making the poor poorer [5][14].

\section{Conclusion}

Artificial Intelligence has grown as an integral part of our daily lives, knowingly or unknowingly. AI has surely made a lot of daily activities easier for human beings, therefore its use and demand are at their peak in almost every possible sector. Machines are capable of performing tasks that consist of using detailed instructions and computational abilities. Tedious and complex tasks are easily performed with the assistance of AI tools. On the other hand, the increased use of AI directly relates to the replacement of the human force. However, the reliability of machines may go wrong if the logic building, knowledge, or any other central aspect is misinterpreted by the machines or while training. There are different opinions on how AI will affect the lives of people in the long run but they have had a significant impact on the daily lives of people since it came into existence. They have various applications in the real world including medical sciences, defence, entertainment, and many more. AI can serve humanity very well in the future and make human life easier if the right measures are taken to develop it and its consequences are thoroughly analyzed.

\section{Declarations}

\subsection{Study Limitations}

This area of research is majorly dependent on support by experts/research studies, organizations, and authorities which makes it is future uncertain to be predicted correctly.

\subsection{Acknowledgments}

The assistance provided by our friends and family throughout the writing of this paper by providing opinions and resources is greatly appreciated. 
Saini et al., Adv. J. Grad. Res.; Vol. 11, Issue 1, pp: 10-22, January 2022

\subsection{Competing Interests}

Authors have no conflict of interest.

\section{How to Cite this Article:}

F. Saini, T. Sharma, and S. Madan, "A Comparative Analysis of Expert Opinions on Artificial Intelligence: Evolution, Applications, and Its Future”, Adv. J. Grad. Res., vol. 11, no. 1, pp. 10-22, Sep. 2021. https://doi.org/10.21467/ajgr.11.1.10-22

\section{References}

[1] J. Shabbir, T. Anwer, “Artificial Intelligence and its Role in Near Future”, Journal of Latex class files, Vol. 14, No. 8, pp.1-9, Apr 2018. https://arxiv.org/abs/1804.01396v1

[2] R. H. Ner, "Evolution and Revolution in Artificial Intelligence" International Journal of Advanced Research in Science, Communication and Technology (IJARSCT), Vol..11, Issue 2, pp.125-129, Nov 2020. https://doi.org/10.48175/IJARSCT-612

[3] A. Murshida A, Chaithra B K, Nishmitha B, P B Pallavi, Raghavendra S, M. Prasanna K, "Survey on Artificial Intelligence" International Journal of Computer Sciences and Engineering, Vol.7, Issue-5, pp.1779-1790, May 2019. https://doi.org/10.26438/ijcse/v7i5.17781790

[4] Y. Dong, J. Hou, N. Zhang, and M. Zhang, "Research on How Human Intelligence, Consciousness, and Cognitive Computing Affect the Development of Artificial Intelligence", Hindawi Complexity, Vol. 2020, pp.1-10, Oct 2020. https://doi.org/10.1155/2020/1680845

[5] Y. Lu, "Artificial intelligence: a survey on evolution, models, applications and future trends", Journal of Management Analytics, Vol. 6, Issue 1, pp.1-29, Feb 2019. https://doi.org/10.1080/23270012.2019.1570365

[6] A. F.S. Borges, F. J.B. Laurindoa, M. M. Spínolaa, R. F. Gonçalvesb, C. A. Mattos, "The strategic use of artificial intelligence in the digital era: Systematic literature review and future research directions", International Journal of Information Management, Vol. 57, pp.1-16, Aug 2020. https://doi.org/10.1016/j.ijinfomgt.2020.102225

[7] A. R, D. Arasa, S. Jamadade "A survey on Artificial Intelligence" International Journal of Engineering Research \& Technology (IJERT), Vol. 6, Issue 13, pp.1-6, Apr 2018.

[8] J. Borana "Applications of Artificial Intelligence \& Associated Technologies" Proceeding of International Conference on Emerging Technologies in Engineering, Biomedical, Management and Science [ETEBMS-2016], pp.64-67, Mar 2016.

[9] H. Lu, Y. Li, M. Chen, H. Kim1 and S. Serikawal "Brain Intelligence: Go beyond Artificial Intelligence", Mobile Networks and Applications, Vol. 23, Issue 2, pp. 368-375, Sept 2017. https://doi.org/10.1007/s11036-017-0932-8

[10] J. Liu, X. Kong, F. Xia, X. Bai, L. Wang, Q. Qing, I. Lee, “Artificial Intelligence in the 21 st Century” IEEE Access, Vol. 6, pp.3440334421, Mar 2018.

[11] J. Shaw, F. Rudzicz, T. Jamieson and A. Goldfarb, “Artificial Intelligence and the Implementation Challenge” Journal Of Medical Internet Research, Vol. 21, Issue 7, Jul 2019.

[12] R. Dasoriya, J. Rajpopat, R. Jamar and M. Maurya, "The Uncertain Future of Artificial Intelligence," 2018 8th International Conference on Cloud Computing, Data Science \& Engineering (Confluence), pp. 458-461, Jan 2018.

[13] S. M. C. Loureiro, J. Guerreiro, I. Tussyadiah, “Artificial intelligence in business: State of the art and future research agenda“, Journal of Business Research, Vol. 129, pp.911-926, May 2021. https://doi.org/10.1016/j.jbusres.2020.11.001

[14] J. Anderson, L. Rainie, and D. A. Luchsinger, “Artificial Intelligence and the Future of Humans", Pew Research Center, Dec 2018.

[15] A. Pannu "Artificial Intelligence and its Application in Different Areas," International Journal of Engineering and Innovative Technology (IJEIT), Vol. 4, Issue 10, pp. 79-84, Apr 2015

[16] S. Sarangi and P. Sharma, "Introduction", Artificial Intelligence-Evolution, Ethics and Public Policy, 2019.

Publish your books with AIJR publisher-

$\checkmark$ Publish with ISBN and DOI.

$\checkmark$ Publish Thesis/Dissertation as Monograph.

$\checkmark$ Publish Book Monograph.

$\checkmark$ Publish Edited Volume/ Book.

$\checkmark$ Publish Conference Proceedings

$\checkmark \quad$ Retain full copyright of your books.

Submit your manuscript at books.aijr.org
Publish your research article in AIJR journals-

$\checkmark \quad$ Online Submission and Tracking

$\checkmark$ Peer-Reviewed

$\checkmark$ Rapid decision

$\checkmark \quad$ Immediate Publication after acceptance

$\checkmark \quad$ Articles freely available online

$\checkmark \quad$ Retain full copyright of your article.

Submit your article at journals.aijr.org 